\title{
Variation of bispectral index in children aged 1-12 years under propofol anesthesia: an observational study
}

\author{
Fang Wang ${ }^{1}$, Jianmin Zhang ${ }^{1}$, Jie Yu', Muyang Tian ${ }^{1}$, Xiaohuan Cui ${ }^{1}$ and Anshi Wu ${ }^{2^{*}}$ (D)
}

\begin{abstract}
Background: The use of the bispectral index (BIS) is widespread in pediatric anesthesia, but few studies have attempted to perform a detailed evaluation of how BIS varies according to age in children under propofol anesthesia. This prospective study aimed to explore the exact relationship between BIS value and the age of 1- to 12-year-old children under propofol anesthesia.

Methods: This study enrolled 165 children $(1<y$ r. $\leq 12)$, scheduled for surgery under anesthesia, and divided them into 11 age groups. Of the 165 participants, 157 completed the study protocol. All patients were anesthetized with propofol for over $30 \mathrm{~s}$. An observation period of $4 \mathrm{~min}$ followed. BIS values were recorded at 0 (immediately after propofol injection), 30, 40, 50, 60, 90, 120, 180, and $240 \mathrm{~s}$ after the injection. BIS values at each time point corresponding to the 11 age groups were compared using repeated measures ANOVA.
\end{abstract}

Results: BIS values significantly differed among the nine time points $(p<0.01)$ as well as among the different age groups $(p<0.01)$ after propofol administration. Post-hoc Bonferroni tests showed a difference in BIS values between groups $1-4(1<y r . \leq 5)$ and groups $5-11(5<y r . \leq 12)$. BIS values were lower in the latter than in the former, from 50 to $240 \mathrm{~s}$. The minimum BIS values in group $1<\mathrm{yr}$. $\leq 5$ and in group $5<\mathrm{yr}$. $\leq 12$ were recorded at $60 \mathrm{~s}$ as $49 \pm 17$ and $35 \pm 14$, respectively.

Conclusions: During propofol anesthesia, the BIS values were closely related to age, which can be divided into two groups: $1<\mathrm{yr}$. $\leq 5$ and $5<\mathrm{yr}$. $\leq 12$. BIS values of the older age group were lower than those of the younger age group at the same time points.

Trial registration: Registration number: chictr-roc-16008630. Registered on 12 June 2016. Retrospectively registered.

Keywords: Propofol, Anesthesia: intravenous, Bispectral index, Children

\section{Background}

Since the implementation of the bispectral index (BIS) as the only means of monitoring the depth of sedation by the American Food and Drug Administration, its application has become increasingly widespread in pediatric anesthesia. Although the reliability of BIS is similar among adults and children over the age of 1 year [1], whether BIS can accurately monitor the depth of sedation in children remains controversial. The BIS algorithm was initially derived from

\footnotetext{
* Correspondence: wuanshi012@sina.com

${ }^{2}$ Department of Anesthesiology, Beijing Chaoyang Hospital, Capital Medical University, No. 8 Gongti South Road, Chaoyang District, Beijing 100020, China Full list of author information is available at the end of the article
}

electroencephalography data obtained from adults under various anesthetic conditions [2]. However, the child's brain is different from that of an adult and is characterized by rapid development. Moreover, the Electroencephalogram (EEG) of children has different manifestations at different ages; thus, whether the BIS value can accurately reflect these changes across growth is directly related to its accuracy for monitoring sedation depth. Recent studies using various volatile and intravenous anesthetic agents have shown that BIS values are linked to the age of children [3-6]. However, most of these studies have involved children over the age of 3 years or traditional age groups; infants (1-12 months of age), toddlers (13-36 months), and 
children (37-144 months), and only indicated that a given BIS value was relevant for a specified age. Few studies have attempted to evaluate the details of BIS variation with age under propofol anesthesia in a pediatric population.

This prospective observational study explored the exact relationship between BIS values and children aged 1-12 years, grouped with one-year intervals, under propofol anesthesia to provide a reliable basis for clinical monitoring.

\section{Methods}

\section{Ethical approval}

This study (Chictr.org.cn identifier: chictr-roc-16,008, 630) was approved by the Ethical Committee of Beijing Children's Hospital, Capital Medical University, Beijing, China (Ethical Committee No 2016-67, Chairperson Prof Tianyou Wang) on 16 May 2016. Written informed consent for participation was obtained from the parents or guardians of all patients.

\section{Study population}

In total, 165 pediatric patients requiring elective surgery, including urological, orthopedic, thoracic, and general surgery, under general anesthesia via intubation or a laryngeal mask, were initially selected. The inclusion criteria were as follows: $1<\mathrm{yr} . \leq 12$, an American Society of Anesthesiologists (ASA) score of I or II, the intraoperative use of total intravenous anesthesia, and the provision of written informed consent by a parent or guardian. The exclusion criteria were as follows: concomitant severe systemic disease; requirement of cranial surgery; long-term use of sedative, hypnotic, or antipsychotic drugs; history of epilepsy; concomitant mental development disorders; and concomitant cardiovascular disease. We divided the included patients into 11 age groups with 15 patients in each group: group $1(1<$ yr. $\leq 2)$, group $2(2<\mathrm{yr} . \leq 3)$, group $3(3<\mathrm{yr} . \leq 4)$, group 4 $(4<$ yr. $\leq 5)$, group $5(5<\mathrm{yr} . \leq 6)$, group $6(6<\mathrm{yr} . \leq 7)$, group $7 \quad(7<\mathrm{yr} . \leq 8)$, group $8(8<\mathrm{yr} . \leq 9)$, group 9 $(9<\mathrm{yr} . \leq 10)$, group $10(10<\mathrm{yr} . \leq 11)$, and group 11 $(11<\mathrm{yr} . \leq 12)$.

\section{Anesthesia protocol}

No children were pre-medicated. Children routinely fasted from food for $\geq 6 \mathrm{~h}$ and from drink for $\geq 4 \mathrm{~h}$ before surgery. Peripheral intravenous access was established for all patients in the hospital ward. In the operating room, a continuous monitor (S/5TM monitor; GE Healthcare, Germany) was used for noninvasive blood pressure (NIBP), pulse oxygen saturation $\left(\mathrm{SpO}_{2}\right)$, heart rate (HR), and electrocardiogram (ECG) monitoring. After oxygen inhalation via a facemask was initiated, general anesthesia was induced by the continuous intravenous injection of 3 $\mathrm{mg} / \mathrm{kg}$ of propofol (1\% propofol in a medium-chain/longchain triglyceride emulsion; Fresenius Kabi Deutschland Gmbh, Germany) for over 30 s, followed by an observation period of $4 \mathrm{~min}$. Routine oxygen inhalation via the facemask was continued during propofol administration, and the lower jaw was gently raised as needed to assist airway management and maintain a blood oxygen saturation of $\geq 97 \%$. At the end of the observation period, $2 \mathrm{mg} / \mathrm{kg}$ of propofol (Fresenius Kabi Deutschland Gmbh, Germany), $2 \mu \mathrm{g} / \mathrm{kg}$ of fentanyl (Yichang Humanwell Pharmaceutical Co., Ltd., Hubei Province, China), and $0.5 \mathrm{mg} / \mathrm{kg}$ of rocuronium (Hameln Pharmaceuticals Gmbh, Germany) were administered for routine induction. The patient was intubated or received a laryngeal mask after a sufficient depth of anesthesia was achieved.

\section{Data acquisition}

BIS (Aspect Medical System, USA) was simultaneously monitored for all patients. The skin over the forehead was cleaned, and the BIS electrode was then placed according to the manufacturers instructions by the anesthesiologist. The BIS monitor requires a self-test before it begins to function. Propofol was injected after signal stabilization, which indicated the BIS value in the absence of anesthesia. The baseline values were recorded immediately after propofol injection was initiated $(0 \mathrm{~s})$. Subsequently, values were recorded at $30,40,50,60,90,120,180$, and $240 \mathrm{~s}$ after the injection.

For all patients, the same anesthesiologist placed all electrodes and administered propofol. Moreover, data for all patients were recorded by the same individual.

\section{Statistical analysis and data handling}

The sample size of 15 cases per group in the present study was generally similar, but not identical, to that of previously published reports $[7,8]$.

BIS values were measured at nine time points in the 11 age groups. IBM SPSS 21.0 (SPSS Inc., Chicago, IL, USA) was used for data analysis. Normally distributed numerical data are expressed as means \pm standard deviations (SDs; $\overline{\mathrm{x}} \pm \mathrm{s}$ ). Repeated measures ANOVA was used to analyze BIS values at multiple time points in groups. A post-hoc Bonferroni test was used to compare differences at same time points in different age groups and at different time points in same groups. A $p$-value of $<0.05$ was considered statistically significant. Data analysis was independently performed by two data scientists.

\section{Results}

From the 165 initially recruited patients, eight were excluded because the electrodes fell off during the observation period; 157 patients were thus included in the analyses. The patient data are presented in Table 1 . 
Table 1 Patient characteristics of 11 groups

\begin{tabular}{|c|c|c|c|c|c|c|c|c|c|c|c|c|}
\hline Groups & 1 & 2 & 3 & 4 & 5 & 6 & 7 & 8 & 9 & 10 & 11 & Total \\
\hline Number of patients & 15 & 15 & 15 & 15 & 13 & 15 & 15 & 15 & 13 & 12 & 14 & 157 \\
\hline Male/Female & $13 / 2$ & $14 / 1$ & $13 / 2$ & $10 / 5$ & $7 / 6$ & $10 / 5$ & $12 / 3$ & $13 / 2$ & $6 / 7$ & $9 / 3$ & $9 / 5$ & $116 / 41$ \\
\hline \multicolumn{13}{|l|}{ Type of surgery } \\
\hline Urology & 11 & 10 & 6 & 7 & 9 & 5 & 5 & 1 & & 3 & 5 & 62 \\
\hline Orthopaedics & 1 & 3 & 9 & 6 & 3 & 7 & 10 & 9 & 9 & 9 & 7 & 73 \\
\hline Other & 3 & 2 & & 2 & 1 & 3 & & 5 & 4 & & 2 & 22 \\
\hline
\end{tabular}

group $1(1<\mathrm{yr} . \leq 2)$, group $2(2<\mathrm{yr} . \leq 3)$, group $3(3<\mathrm{yr} . \leq 4)$, group $4(4<\mathrm{yr} . \leq 5)$, group $5(5<\mathrm{yr} . \leq 6)$, group $6(6<\mathrm{yr} . \leq 7)$, group $7(7<\mathrm{yr}$. $\leq 8)$, group $8(8<\mathrm{yr} . \leq$ $9)$, group $9(9<\mathrm{yr} . \leq 10)$, group $10(10<\mathrm{yr} . \leq 11)$, and group $11(11<\mathrm{yr} . \leq 12)$

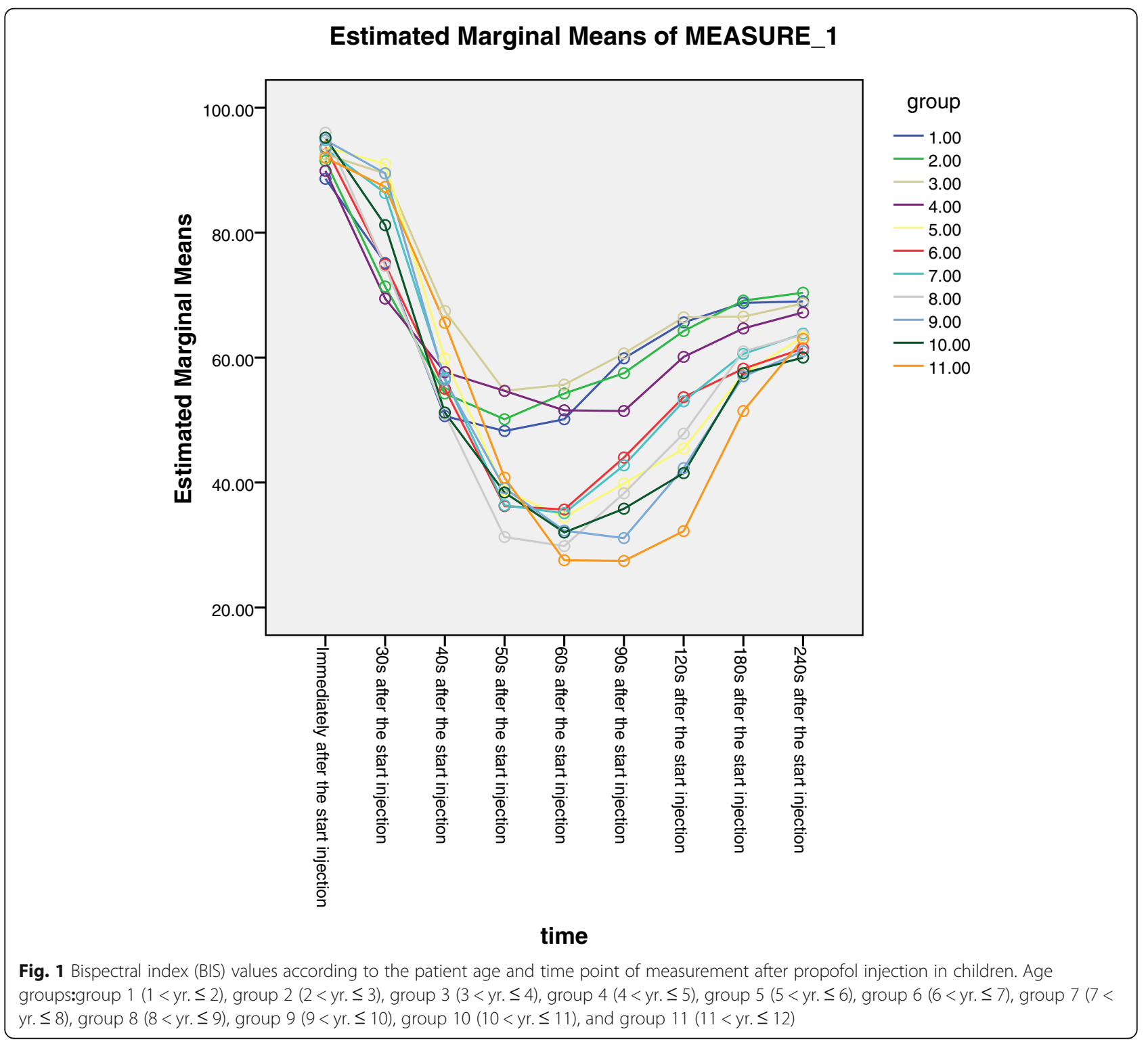


Repeated measures ANOVA showed that BIS values significantly differed among the nine time points $(P<0.01)$ as well as the different age groups $(P<0.01)$ after propofol administration. The post-hoc Bonferroni test showed a difference in BIS values between groups $1-4 \quad(1<\mathrm{yr} . \leq 5)$ and groups $5-11(5<$ yr. $\leq 12)$ (Fig. 1$)$.

The patients were reclassified for further analysis: group $1<\mathrm{yr} . \leq 5$, which included the 60 patients in groups $1-4$, and group $5<\mathrm{yr} . \leq 12$, which included the 97 patients in groups $5-11$. Table 2 presents the BIS values at each time point for group $1<\mathrm{yr} . \leq 5$ and group $5<$ yr. $\leq 12$. In both groups, the baseline BIS values (immediately after the start of injection; $0 \mathrm{~s}$ ) differed significantly from the values at the subsequent eight time points, from 30 to $240 \mathrm{~s}(p<0.01)$. The BIS values between the two groups were significantly different at each of the following time points: $50,60,90,120,180$, and $240 \mathrm{~s}(p<0.05)$. The BIS values in group $5<\mathrm{yr} . \leq 12$ were significantly lower than those in group $1<\mathrm{yr} . \leq 5$ at each time point from 50 to $250 \mathrm{~s}(p<0.05)$. The minimum observed BIS values for group $1<\mathrm{yr} . \leq 5$ and group $5<\mathrm{yr} . \leq 12$ were recorded at $60 \mathrm{~s}: 49 \pm 17$ and $35 \pm 14$, respectively. In group $5<$ yr. $\leq 12$, BIS values at 40,50 , and $60 \mathrm{~s}$ were significantly different from one another $(p<0.01)$; group $1<\mathrm{yr} . \leq 5$ did not feature significant differences among these time points $(p>0.05)$. Figure 2 shows the changes in BIS values over time in groups $1<\mathrm{yr} . \leq 5$ and $5<\mathrm{yr}$. $\leq 12$. BIS values in the two patient groups exhibited a rapid decrease to their

Table 2 BIS values at each time point in Group $1<\mathrm{yr}$. $\leq 5$ and Group $5<\mathrm{yr} . \leq 12(\bar{x} \pm \mathrm{s})$

\begin{tabular}{lll}
\hline time/ & BIS & \\
\cline { 2 - 3 } 0 & Group $1<y r . \leq 5$ & Group $5<y r . \leq 12$ \\
30 & $91 \pm 7$ & $94 \pm 5$ \\
40 & $75 \pm 20^{*}$ & $82 \pm 21^{*}$ \\
50 & $57 \pm 21^{* c d}$ & $58 \pm 25^{* e}$ \\
60 & $52 \pm 18^{* a c}$ & $37 \pm 16^{* a f}$ \\
90 & $49 \pm 17^{* a c}$ & $35 \pm 14^{* a f}$ \\
120 & $55 \pm 13^{* a c d}$ & $37 \pm 11^{* a f}$ \\
180 & $63 \pm 8^{* a d}$ & $46 \pm 14^{* a}$ \\
240 & $66 \pm 5^{* a d}$ & $57 \pm 8^{* a e}$ \\
\hline$P<0.05$, values of BIS were compad bedw th grop $1<y r \leq 5$ and
\end{tabular}

${ }^{a} P<0.05$, values of BIS were compared between the group $1<\mathrm{yr} . \leq 5$ and the group $1<\mathrm{yr} . \leq 2$ at the same time point

${ }^{*} P<0.01$, BIS values with superscript * compared with BIS values at 0 s time point

c $P<0.05$, BIS values with superscript $c$ compared with BIS values at the other time point of group $1<\mathrm{yr} . \leq 5$

d $P<0.05$, BIS values with superscript $d$ compared with BIS values at the other time point of group $1<\mathrm{yr} . \leq 5$

e $P<0.05$, BIS values with superscript e compared with BIS values at the other time point of group $5<\mathrm{yr} . \leq 12$

${ }^{f} P<0.05$, BIS values with superscript $d$ compared with BIS values at the other time point of group $5<\mathrm{yr} . \leq 12$ minimum values from 0 to $60 \mathrm{~s}$, followed by an obvious trend towards an increase. Changes in BIS values were more obvious in group $5<\mathrm{yr} . \leq 12$ than in group $1<\mathrm{yr} . \leq 5$, with the former demonstrating a precipitous decrease between 40 and $60 \mathrm{~s}$.

\section{Time points}

0 : immediately after the start of injection.

$30,40,50,60,90,120,180$, and $240 \mathrm{~s}$ after the start of propofol injection.

\section{Discussion}

In this study, BIS value changes were studied in children aged 1-12 years, who were grouped according to age, under propofol anesthesia. We found that BIS values showed a similar trend at each time point after propofol injection in the 11 groups, but BIS values differed according to age groups. Our findings differed from those reported previously.

The pediatric nervous system differs from that of adults in that it is characterized by rapid development; hence, typical pediatric EEGs are more variable than adult EEGs. It has been reported that EEG signals of children mature and stabilize at approximately 12 years of age [9]. Furthermore, while previous reports have studied the relationship between BIS values and propofol after age grouping, most adopted the traditional age grouping method, with the youngest patients being over 3 years old $[4,8,10,11]$. While relevant studies on children younger than 3 years of age have been conducted, the age classification that they employed was inconsistent and lacked an appropriate justification [1, 7]. Changes in EEG signals during the growth and development of young children occur in a short period of time; BIS values may thus feature a similarly significant change and thereby impact the accuracy of BIS monitoring. This study therefore divided children aged 1-12 years into 11 groups with one-year intervals to determine whether BIS values differed among the groups stratified by age.

As the utility of BIS for monitoring the sedative depth of propofol anesthesia in children has been established, propofol is widely used for both sedation and general anesthesia in children [12]. However, BIS monitoring is easily disrupted by the administration of other drugs, especially muscle relaxants. Because BIS features frontal electromyography (EMG) as a weighted parameter, the frontal EMG overlaps with the EEG at a frequency of $30-50 \mathrm{~Hz}$, and the interference of frontal EMG can thus be excluded at frequencies lower than $47 \mathrm{~Hz}$. However, after the application of muscle relaxants, BIS may decrease due to the drop in the components of the frontal EMG, and the monitoring of sedation depth may be disrupted. The BIS value can reportedly reduce to 33 when muscle relaxants are administered 


\section{Estimated Marginal Means of MEASURE_1}

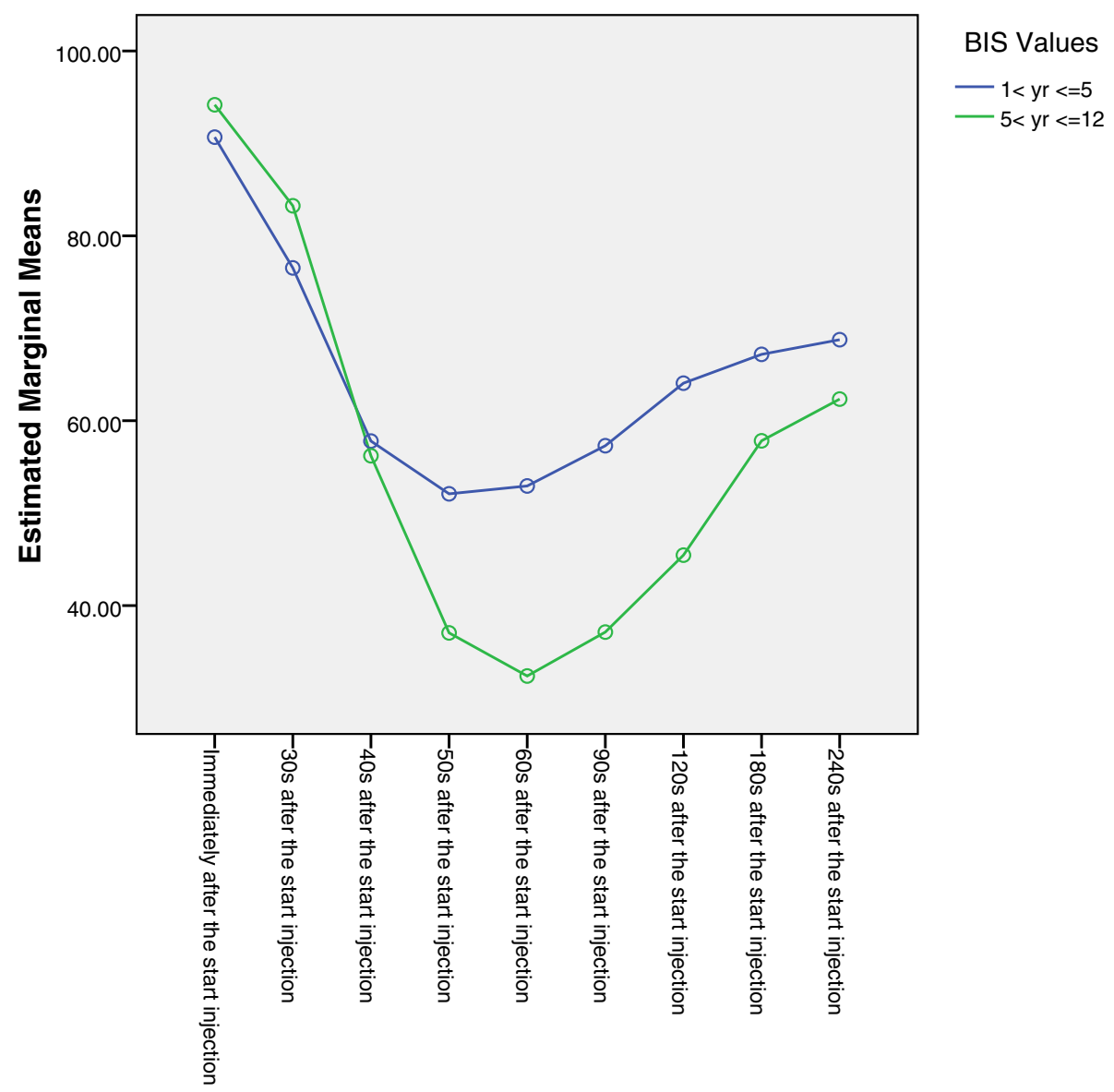

time

Fig. 2 Changes in bispectral index (BIS) values over time in group $1<y \mathrm{r}$. $\leq 5$ and group $5<y \mathrm{r}$. $\leq 12$. Group $1<\mathrm{yr}$. $\leq 5$ : the 60 paediatric patients in groups 1-4 (group 1: $1<y r . \leq 2$; group 2: $2<y r . \leq 3$; group 3: $3<y r . \leq 4$; group 4: $4<y r . \leq 5$ ), who were combined because of the lack of significant differences in BIS values. Group 5<yr. $\leq 12$ : the 97 paediatric patients in groups 5-11 (group 5: $5<\mathrm{yr}$. $\leq 6$; group 6: $6<\mathrm{yr}$. $\leq 7$; group 7: $7<\mathrm{yr}$. $\leq$; group 8: $8<\mathrm{yr}$. $\leq$ 9; group 9: $9<\mathrm{yr}$. $\leq 10$; group 10: $10<\mathrm{yr}$. $\leq 11$; group 11: $11<\mathrm{yr}$. $\leq 12$ ), who were combined because of the lack of significant differences in BIS values

to patients who are fully awake [13]. Whether opioids have an impact on the BIS value remains controversial: although many studies have reported that opioids do not affect BIS values [14, 15], Minto and colleagues [16] have described age-dependent EEG modification when remifentanil was administered to adults and found that changes in BIS values with an attendant loss of verbal command or eyelash reflex were more common in patients who received remifentanil combined with propofol than propofol alone [17, 18]. Therefore, this study administered a single propofol to eliminate the effect of the combined drugs on BIS value and improve the accuracy of the research results.

Previous literature has shown that BIS values for children under propofol anesthesia are significantly associated with age [1]; however, prior studies have only shown that BIS accuracy is better in children over the age of 1 year. In this study, the results of detailed age grouping indicated that BIS values in the 11 groups showed a similar trend in BIS: an initial decrease followed by an increase. However, the changes of BIS values at the same time points differed significantly across age. Moreover, we identified that such significant differences could be identified between two composite groups: $1-4$ and $5-11$. The results suggest that age persists as an important factor, affecting BIS in children over 1 year of age, and anesthesiologists must pay attention to this difference when monitoring sedation depth.

The minimum BIS values of $49 \pm 17$ and $35 \pm 14$ in group $1<\mathrm{yr} . \leq 5$ and group $5<\mathrm{yr} . \leq 12$, respectively, were observed at $60 \mathrm{~s}$. Generally consistent with reports of the peak effect time being at $1.6 \mathrm{~min}$ (1-2.4 min) after the single-bolus intravenous injection of 
propofol [19]. From this finding we presume that BIS is sensitive to changes in blood propofol concentration and similar result had been reported in the previous literature [20]. The change of BIS values in group $5<$ yr. $\leq 12$ was more rapid than that in group $1<\mathrm{yr} . \leq 5$; the former featured a precipitous decrease between 40 and $60 \mathrm{~s}$, and the change in BIS over the same period was not statistically significant in group $1<\mathrm{yr} . \leq 5$. In addition, the BIS values at each time point from 50 to $240 \mathrm{~s}$ showed consistent statistically significant differences between group $1<\mathrm{yr} . \leq 5$ and group $5<\mathrm{yr} . \leq 12$; the BIS values in group $5<\mathrm{yr} . \leq 12$ were all lower than those in group $1<\mathrm{yr} . \leq 5$. This result is consistent with those of previous reports that found BIS values in older age groups to be lower than those in younger age groups [7, 8]. These findings imply that anesthesiologists should appropriately reduce the single injection dose of propofol for children over 5 years old in order to avoid BIS values of less than 40.

There were several limitations of this study that were important to note. First, this study did not provide hemodynamics data at each time point. The current study was designed to observe the change of BIS value after single dose of propofol injection and the 10-s interval between each observation time point was narrow, it may be difficult to measure non-invasive blood pressure at each time points and we also considered that even if we did measure it, Moreover, cuff blood pressure measurement may be considered as one of stimulations during the observation period which may interfere with the sedative state of the child. But heart rate and pulse oxygen saturation were continuously monitored during the whole procedure. Secondly, the current study did not provide information of clinical parameters at each time points, the correlation between BIS value and clinical consciousness was therefore not evaluated in this study. This was the initial observational study for BIS value and propofol injection in different age groups and the correlation BIS values and clinical signs of sedative should be assessed in our future studies.

\section{Conclusions}

Our study indicates that BIS values showed a close relationship to two age groups: $1<$ yr. $\leq 5$ and $5<$ yr. $\leq 12$ during the administration of propofol anesthesia. BIS values of the older age group were lower than those of the younger age group at the same time points. This study suggests that attention should be paid to changes in children younger and older than 5 years when BIS monitoring is applied to children aged $1-12$ years.

Abbreviations

BIS: Bispectral index; EEG: Electroencephalogram

\section{Authors' contributions}

All authors have read and approved the final version of this manuscript. AW contributed to the overall study design, data analysis, and revised the paper. FW helped design the study, performed the experiments, collected and analyzed data, and wrote the manuscript. JZ helped design the study and revised the manuscript. JY, MT and XC had performed the experiments, collected data. All authors had read and approved the final manuscript.

\section{Funding}

This study was supported by Beijing Municipal Science \& Technology Commission, PR China [grant number: Z161100000516142]. The funding agent plays no role in data collection, or data analyses.

\section{Availability of data and materials}

We declared that materials described in the manuscript, including all relevant raw data, will be freely available to any scientist wishing to use them for non-commercial purposes, without breaching participant confidentiality by contacting the corresponding author.

\section{Ethics approval and consent to participate}

This study was performed according to the Declaration of Helsinki after the approval of the Ethical Committee of Beijing Children's Hospital of Capital Medical University, Beijing, China (Ethical Committee No 2016-67) on 16 May 2016. Written informed consent for participation was obtained from the parents or guardians of all patients.

Consent for publication

Not applicable.

\section{Competing interests}

The authors declare that they have no competing interests.

\section{Author details}

${ }^{1}$ Department of Anesthesiology, Beijing Children's Hospital, Capital Medical University, National Center for Children's Health, No. 56 Nanlishi Road, Beijing 100045, China. ²Department of Anesthesiology, Beijing Chaoyang Hospital, Capital Medical University, No. 8 Gongti South Road, Chaoyang District, Beijing 100020, China.

Received: 24 March 2019 Accepted: 29 July 2019

Published online: 07 August 2019

\section{References}

1. Jeleazcov C, Schmidt J, Schmitz B, et al. EEG variables as measures of arousal during propofol anaesthesia for general surgery in children: rational selection and age dependence. Br J Anaesth. 2007;99:845-54.

2. Sebel PS, Lang E, Rampil IJ, et al. A multicenter study of bispectral electroencephalogram analysis for monitoring anesthetic effect. Anesth Analg. 1997;84:891-9.

3. Wodey E, Tirel O, Bansard JY, et al. Impact of age on both BIS values and EEG bispectrum during anaesthesia with sevoflurane in children. $\mathrm{Br} J$ Anaesth. 2005;94:810-20.

4. Tirel O, Wodey $E$, Harris R, et al. The impact of age on bispectral index values and EEG bispectrum during anaesthesia with desflurane and halothane in children. $\mathrm{Br} J$ Anaesth. 2006;96:480-5.

5. Wallenborn J, Kluba K, Olthoff D. Comparative evaluation of bispectral index and Narcotrend index in children below 5 years of age. Paediatr Anaesth. 2007;17:140-7.

6. Tirel O, Wodey $\mathrm{E}$, Harris $\mathrm{R}$, et al. Variation of bispectral index under TIVA with propofol in a paediatric population. Br J Anaesth. 2008;100:82-7.

7. Zhang JM, Wang F, Xin Z, et al. Treatment of different-aged children under bispectral index monitoring with intravenous anesthesia with propofol and remifentanil. Eur Rev Med Pharmacol Sci. 2015;19:64-9.

8. Liu JS, Zhang JM, Yun Y. Variation of bispectral index monitoring in pediatric patients undergoing propofol-remifentanil anesthesia. Eur J Anaesthesiol. 2008:25:821-5.

9. Eisermann M, Kaminska A, Moutard ML, et al. Normal EEG in childhood: from neonates to adolescents. Neurophysiol Clin. 2013;43:35-65.

10. Louvet N, Rigouzzo A, Sabourdin N, et al. Bispectral index under propofol anesthesia in children: a comparative randomized study between TIVA and TCl. Paediatr Anaesth. 2016 Sep;26(9):899-908. 
11. Fuentes R, Cortínez I, Ibacache M, et al. Propofol concentration to induce general anesthesia in children aged 3-11 years with the Kataria effect-site model. Paediatr Anaesth. 2015 Jun;25(6):554-9.

12. Powers KS, Nazarian EB, Tapyrik SA, et al. Bispectral index as a guide for titration of propofol during procedural sedation among children. Pediatrics. 2005 Jun;115(6):1666-74.

13. Messner M, Beese U, Romstock J, et al. The bispectral index declines during neuromuscular block in fully awake persons. Anesth Analg. 2003;97:488-91.

14. Schmidt GN, Bischoff $P$, Standl T, et al. Narcotrend, bispectral index, and classical electroencephalogram variables during emergence from propofol/remifentanil anesthesia. Anesth Analg. 2002;95:1324-30.

15. Wang LP, McLoughlin P, Paech MJ, et al. Low and moderate remifentanil infusion rates do not alter targetcontrolled infusion propofol concentrations necessary to maintain anesthesia as assessed by bispectral index monitoring. Anesth Analg. 2007:104:325-31.

16. Minto CF, Schnider TW, Egan TD, et al. Influence of age and gender on the pharmacokinetics and pharmacodynamics of remifentanil. I. Model development. Anesthesiol. 1997;86:10-23.

17. Struys MM, Vereecke H, Moerman A, et al. Ability of the bispectral index autoregressive modelling with exogenous inputderived auditory evoked potentials, and predicted propofol concentrations to measure patient responsiveness during anesthesia with propofol and remifentanil. Anesthesiology. 2003;99:802-12.

18. Lysakowski C, Dumont L, Pelle'grini M, et al. Effects of fentanyl, alfentanil, remifentanil and sufentanil on loss of consciousness and bispectral index during propofol induction of anaesthesia. Br J Anaesth. 2001:86:523-7.

19. Sepulveda PO, Mora X. Reevaluation of the time course of the effect of propoful described with the Schnider pharmacokinetic model. Rev Esp Anestsiol Reanim. 2012;59:542-8.

20. Doi M, Gajraj RJ, Mantzaridis H, et al. Relationship between calculated blood concentration of propoful and electrophysiological variables during emergence from anaesthesia: comparision of bispectral index, spectral edge frequency, median frequency and auditory evoked potential index. Br J Anaesth. 1997;78:180-4.

\section{Publisher's Note}

Springer Nature remains neutral with regard to jurisdictional claims in published maps and institutional affiliations.

Ready to submit your research? Choose BMC and benefit from:

- fast, convenient online submission

- thorough peer review by experienced researchers in your field

- rapid publication on acceptance

- support for research data, including large and complex data types

- gold Open Access which fosters wider collaboration and increased citations

- maximum visibility for your research: over $100 \mathrm{M}$ website views per year

At $\mathrm{BMC}$, research is always in progress.

Learn more biomedcentral.com/submissions 